\title{
An unusual case with genetic transition in a rehabilitation clinic: Troyer syndrome
}

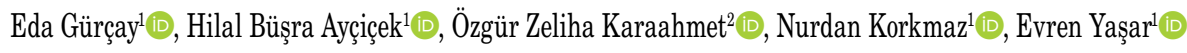 \\ 1Department of Physical Medicine and Rehabilitation, University of Health Sciences, Gaziler Physical Therapy and Rehabilitation Training and Research Hospital, Ankara, Turkey \\ ${ }^{2}$ Department of Physical Medicine and Rehabilitation, University of Health Sciences, Ankara Dışkapı Yıldırım Beyazıt Training and Research Hospital, Ankara, Turkey
}

Received: August 08, 2020 Accepted: December 26, 2020 Published online: May 25, 2021

Hereditary spastic paraplegia (HSP) refers to rare neurogenetic disorders, characterized by progressive muscle weakness and spasticity of the lower limbs. A nucleotide deletion in the spartin gene (SPG20) is the origin of autosomal recessive Troyer syndrome, a complex form of HSP. ${ }^{[1]}$

The content of this paper is to discuss an unusual case, namely Troyer syndrome, with spastic paraparesis, distal amyotrophy, short stature due to growth failure, dysarthria, emotional lability, and low intelligence and rehabilitation management of this extremely rare disorder.

A 12-year-old boy was admitted to our rehabilitation clinic with a history of first speech at the end of nine months and first walking at 18 months with frequent fallings. A genetic mutation was detected in the SPG20 gene (p.Met122ValfsTer2), encoding the spartin. Parents were first-degree cousins who were healthy. Physical examination showed a short stature (percentile values $<3 \%$ ). He understood simple commands and could speak a few words in the dysarthric form. He had a low nasal bridge, short neck, and a toothy smile. There was apparently considerable weakness of the lower extremities and distal amyotrophy. Gait was wide-based and in the spastic pattern. He was able to ambulate with knees in recurvatum, feet in planovalgus, and bilateral ankle-foot orthosis. The results of laboratory investigations, screening tests for metabolic diseases, electromyography, and cranial magnetic resonance imaging revealed no abnormalities. The patient was scheduled for a rehabilitation program consisting of limb muscle strengthening exercises, balance exercises, gait training, and aquatic interventions. For a more steady and balanced gait pattern, an appropriate walker was prescribed. While the patient was discharged, he was able to walk safely in a stable manner and for a long distance. A written informed consent was obtained from the parent of the patient.

Individuals with HSP among the Turkish population have usually skeletal abnormalities, spasticity, and distal muscle atrophy. Short stature is considered a typical sign of affected individuals. ${ }^{[2]}$ The Amish population shows dysarthria, hyperreflexia, spasticity of the lower limbs, distal muscular atrophy with normal electromyography. ${ }^{[3]}$ Motor development is usually delayed, and they begin to walk between 16 and 24 months with different gait patterns. Mental delay may occur at an early period, although affected patients usually do not show any signs of retardation. ${ }^{[4]}$ Our case showed clinical overlap such as short stature, decreased muscle tone in the arms, spastic paraparesis, motor, speech, and cognitive delays. 
To the best of our knowledge, there is no report in the literature discussing the rehabilitation and management approach for these patients. The scheduled and applied rehabilitation program can provide positive contributions in terms of posture and ambulation on discharge. This case may, thus, provide additional data to the unresolved, complicated forms of HSP and guide cases to be treated in rehabilitation clinics in the near future.

\section{Declaration of conflicting interests}

The authors declared no conflicts of interest with respect to the authorship and/or publication of this article.

\section{Funding}

The authors received no financial support for the research and/or authorship of this article.

\section{REFERENCES}

1. Fink JK. Hereditary spastic paraplegia overview. Gene Reviews. 2000. Last Update: 2014. Available at: https://www. ncbi.nlm. nih.gov/books/NBK1509/.

2. Manzini MC, Rajab A, Maynard TM, Mochida GH, Tan WH, Nasir R, et al. Developmental and degenerative features in a complicated spastic paraplegia. Ann Neurol 2010;67:516-25.

3. Proukakis C, Cross H, Patel H, Patton MA, Valentine A, Crosby AH. Troyer syndrome revisited. A clinical and radiological study of a complicated hereditary spastic paraplegia. J Neurol 2004;251:1105-10.

4. Neuhäuser G, Wiffler C, Opitz JM. Familial spastic paraplegia with distal muscle wasting in the Old Order Amish; atypical Troyer syndrome or "new" syndrome. Clin Genet 1976;9:315-23. 